\title{
Field susceptibility of a composite system: application to van der Waals dispersive interactions inside a finite line of physisorbed atoms
}

\author{
Christian Girard \\ Laboratoire de Physique Moléculaire UA CNRS 772, Université de Franche Comté, 25030 Besançon Cedex, France
}

\begin{abstract}
Alain Dereux
Institute for Studies in Interface Sciences, Facultés Universitaire Notre-Dame de la Paix, 61, rue de Bruxelles, B-5000 Namur, Belgium
\end{abstract}

and

Olivier J.F. Martin

IBM Research Division, Zurich Research Laboratory, Säumerstrasse 4, CH-8803 Rüschlikon, Switzerland

Received 5 March 1993; accepted for publication 15 June 1993

\begin{abstract}
The growing interest in the study of natural or artificial nanoscale structures stabilized by a corrugated surface calls for specific models adapted to the awkward symmetry of such systems. In this work the field susceptibility of a system composed of a finite number of micro-systems interacting with a solid surface is derived from a Dyson's type equation. The many-body character of the interactions between each particle, including reflection with the solid surface, is taken into account by a self-consistent procedure. We show that the calculation of this susceptibility provides a good basis to obtain the van der Waals dispersion energy inside a finite line of physisorbed atoms. We also discuss the possibility of applying this method to study optical energy transfer in complex systems.
\end{abstract}

\section{Introduction}

The field susceptibility $S\left(r, r^{\prime}, \omega\right)$ of a material system reveals how a dipolar source field is modified at the proximity of the surface limiting this system [1-3]. In a general quantum description, this responsc function can be expressed in terms of the matrix elements of the field operator associated with the material system [1-5]. The deduction of the response field of the solid to a fluctuating dipole moment [5-10] is another way of deriving this susceptibility.

In the particular case of a solid limited by a perfectly planar surface, various theoretical methods have been developed to derive this dyadic tensor. The analytical form of $S\left(r, r^{\prime}, \omega\right)$ depends on the nature of the surface investigated. To treat a crystallographic face of an ionic crystal, a discrete atomic representation of the solid structure must be adopted. For a metallic surface displaying mesoscopic roughness, this susceptibility can be modeled from a continuous description of the matter.

In surface physics the use of the field-susceptibility concept has proved to be a very fruitful way to understand various physical mechanisms occurring in physisorption experiments. For example, in the case of solid-gas interactions, MacLachlan demonstrated that the field susceptibility associated with the substrate is the fundamental quantity for describing the dispersion energy between adsorbed species and 
solids [1,2].The trace of this dyadic tensor $S\left(\boldsymbol{r}, \boldsymbol{r}^{\prime}, \omega\right)$ when contracted with the dynamical polarizability $\alpha_{\mathrm{a}}(\omega)$ of the adsorbate gives the first-order dispersion energy [9] after integration over imaginary frequencies (i $u$ )

$$
U\left(\boldsymbol{R}_{\mathrm{a}}\right)=-\frac{\hbar}{2 \pi} \int_{0}^{\infty} \operatorname{Tr}\left[\alpha_{\mathrm{a}}(\mathrm{i} u) \cdot \boldsymbol{S}\left(\boldsymbol{R}_{\mathrm{a}}, \boldsymbol{R}_{\mathrm{a}}, \mathrm{i} u\right)\right] \mathrm{d} u .
$$

In this equation $\boldsymbol{R}_{\mathrm{a}}$ represents the location of the adsorbate with respect to the surface.

More recently, a generalized formulation of this description was developed by Linder and Rabenold [3,4] and by Agarwal [5]. Different improvements such as spatial dispersion effects [7,8], mesoscopic roughness [9] and atomic corrugations [10] were included in other contributions.

Furthermore, as described in recent papers, the field-susceptibility technique also appears to be efficient for treating the problem of optical energy transfer in complex systems $[11,12]$ (near field optics, nanometer scale confinement of optical fields near surfaces, etc.). The theoretical description of low symmetry interacting systems occurring in scanning near field optical microscopy (probe tip + corrugated sample) is easier with a formalism avoiding matching boundary conditions. As described in ref. [11], the retarded field-susceptibility method offers this opportunity. Furthermore, this technique allows thus to treat both the fundamental (van der Waals) interactions and excited (optics) states of the electromagnetic field.

More recently, the growing interest in the study of nanoscale structures (natural or artificial) stabilized by a corrugated surface requires specific models adapted to the low symmetry of such systems. For example, the recent work of Figler et al. successfully illustrates the actual capability for creating simple atomic or molecular arrays physisorbed on metallic surfaces [13,14]. Finite lines of xenon atoms physisorbed on the $\mathrm{Ni}(110)$ surface have been aligned with the probe tip of a scanning tunneling microscope.

In this paper the field susceptibility of a system composed of a finite number of micro-systems (atoms, molecules, small metallic aggregates, etc.) deposited on a substrate is derived from a Dyson's type equation. The many-body character of the interactions between each particle, including multiple reflections with the substrate, are taken into account by a fully self-consistent procedure (cf. section 2 ). We then show in section 3 that the calculation of this susceptibility provides a good basis for obtaining the van der Waals dispersive energy inside a finite line of physisorbed atoms.

\section{Field susceptibility of a composite system}

\subsection{Model and basic equations}

We consider in this section the problem of the electromagnetic response of a set of $n$ micro-systems (or particles) deposited on the surface of a solid body (see fig. 1). The Fourier component at frequency $\omega$ of the electric field generated by a fluctuating dipole source $m_{0}(\omega)$ located above this system at the position $\boldsymbol{r}_{0}=\left(x_{0}, y_{0}, z_{0}\right)$ is given by

$$
e_{0}(r, \omega)=s_{0}\left(r, r_{0}, \omega\right) \cdot m_{0}(\omega)
$$

where the dyadic tensor $s_{0}\left(r, r_{0}, \omega\right)$ represents the field susceptibility in the presence of the substrate alone. Various analytical forms for this response function are given explicitly in ref. [15] and in section 3 .

In presence of the external field $e_{0}(r, \omega)$ the micro-systems lying on the surface acquire fluctuating dipole moments which are also perturbated by the surrounding dielectric due to the other particles. The 


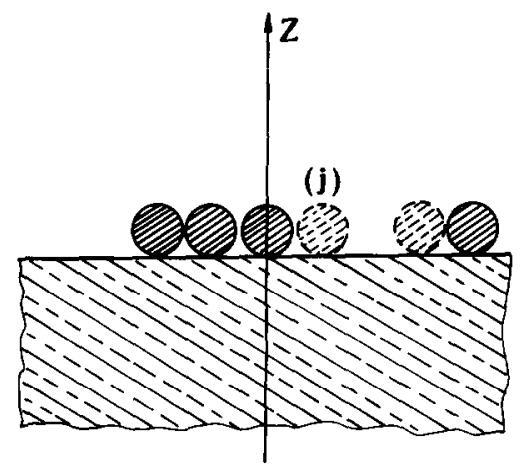

Fig. 1. Schematic illustration of an ensemble of $(n)$ particles deposited on a surface. Each micro-system is located at a position $r_{i}$ with respect to the absolute frame.

self-consistent electric field perturbated by the presence of $\boldsymbol{m}_{0}(\omega)$ verifies the following implicit Lippmann-Schwinger equation [16]

$$
E(r, \omega)=s_{0}\left(r, r_{0}, \omega\right) \cdot m_{0}(\omega)+\iint s_{0}\left(r, r^{\prime}, \omega\right) \cdot \chi\left(r^{\prime}, r^{\prime \prime}, \omega\right) \cdot E\left(r^{\prime \prime}, \omega\right) \mathrm{d} r^{\prime} \mathrm{d} r^{\prime \prime}
$$

In this integral equation, the response function $\chi\left(r^{\prime}, r^{\prime \prime}, \omega\right)$ defines the linear susceptibility associated with the set of micro-systems. We can write this quantity as a sum of the individual susceptibilities characterizing the dynamical response of each particle

$$
\chi\left(r^{\prime}, r^{\prime \prime}, \omega\right)=\sum_{j=1}^{n} \chi_{j}\left(\boldsymbol{r}^{\prime}, \boldsymbol{r}^{\prime \prime}, \omega\right)
$$

where the subscript $j$ defines the location of a particle above the substrate. Within the dipolar approximation, one can write

$$
\chi_{j}\left(r^{\prime}, r^{\prime \prime}, \omega\right)=\alpha_{j}(\omega) \delta\left(r^{\prime}-r_{j}\right) \delta\left(r^{\prime \prime}-r_{j}\right) .
$$

In this relation, $r_{j}$ and $\alpha_{j}(\omega)$ define the position and the polarizability of the $j$ th particle. From the relations (4) and (5), the integral eq. (3) can be expressed in terms of effective fields $E\left(r_{j}, \omega\right)$ existing at each position $j$ occupied by the particles

$$
E(r, \omega)=s_{0}\left(r, r_{0}, \omega\right) \cdot m_{0}(\omega)+\sum_{j=1}^{n} s_{0}\left(r, r_{j}, \omega\right) \cdot \alpha_{j}(\omega) \cdot E\left(r_{j}, \omega\right) .
$$

The solution of eq. (6) requires a self-consistent step which is easily performed thanks to the finite number $n$ of discretization points $\left\{\boldsymbol{r}_{j}\right\}$ which coincides with the number of particles. Indeed the determination of the set of vectors $\left\{\boldsymbol{E}\left(\boldsymbol{r}_{j}, \boldsymbol{\omega}\right)\right\}$ can be performed by a standard linear algebra procedure. Thus by putting $r=r_{i}$ in eq. (6) one obtains a matrix equation to be solved numerically by

$$
F(\omega)=A(\omega) \cdot F_{0}(\omega),
$$

where the symbol - indicates a total contraction on both Cartesian indexes and positions of micro-systems. Moreover $F(\omega)$ and $F_{0}(\omega)$ are two super-vectors defined by

$$
F(\omega)=\left\{E\left(r_{1}, \omega\right) ; E\left(r_{2}, \omega\right) ; \cdots ; E\left(r_{n}, \omega\right)\right\}
$$


and

$$
F_{0}(\omega)=S_{0}^{t}\left(r_{0}, \omega\right) \cdot m_{0}(\omega)
$$

where $S_{0}^{\mathrm{t}}\left(\boldsymbol{r}_{0}, \omega\right)$ is a super-tensor given by

$$
S_{0}^{t}\left(r_{0}, \omega\right)=\left\{s_{0}\left(r_{1}, r_{0}, \omega\right) ; s_{0}\left(r_{2}, r_{0}, \omega\right) ; \cdots ; s_{0}\left(r_{n}, r_{0}, \omega\right)\right\}
$$

In eq. (6), the $(3 n \times 3 n)$ matrix $A$ is built from different components taken by the field susceptibility $s_{0}$ for all possible couples of position vectors $\left\{\boldsymbol{r}_{i}, \boldsymbol{r}_{j}\right\}$

$$
A(\omega)=[1-B(\omega)]^{-1}
$$

where I represents the identity tensor and $\mathrm{B}(\omega)$ is the $(3 n \times 3 n)$ matrix defined by

$$
B(\omega)=\left(\begin{array}{cccc}
\alpha_{1}(\omega) \cdot s_{0}\left(r_{1}, r_{1}, \omega\right) & \alpha_{2}(\omega) \cdot s_{0}\left(r_{1}, r_{2}, \omega\right) & \ldots & \alpha_{n}(\omega) \cdot s_{0}\left(r_{1}, r_{n}, \omega\right) \\
\alpha_{1}(\omega) \cdot s_{0}\left(r_{2}, r_{1}, \omega\right) & \alpha_{2}(\omega) \cdot s_{0}\left(r_{2}, r_{2}, \omega\right) & \ldots & \alpha_{n}(\omega) \cdot s_{0}\left(r_{2}, r_{n}, \omega\right) \\
\ldots & \ldots & \ldots & \ldots \\
\ldots & \ldots & \ldots & \ldots \\
\alpha_{1}(\omega) \cdot s_{0}\left(r_{n}, r_{1}, \omega\right) & \alpha_{2}(\omega) \cdot s_{0}\left(r_{n}, r_{2}, \omega\right) & \ldots & \alpha_{n}(\omega) \cdot s_{0}\left(r_{n}, r_{n}, \omega\right)
\end{array}\right)
$$

This matrix contains all the dynamical and structural information about the composite system. The diagonal terms describe the direct coupling between each micro-system and the substrate, while the off-diagonal terms are responsible for the substrate-mediated interactions between the particles deposited on the surface.

\subsection{Field susceptibility of a small number of physisorbed particles}

When the size of the dynamical square matrix $A(\omega)$ remains reasonable $(n \leq 50)$, it is possible to compute accurate numerical solutions for the effective field distribution contained in the super-vector $F(\omega)$. It is then a simple matter to obtain a general expression for the response field $\boldsymbol{E}(\boldsymbol{r}, \omega)$ defined in eq. (6). The determination of $\boldsymbol{E}(\boldsymbol{r}, \boldsymbol{\omega})$ is usually sufficient in optics. In the context of van der Waals interaction the knowledge of the field susceptibility of the adsorbed species is required (cf. eq. (1)). An additional computational step is thus needed. Substitution of eqs. (7) and (9) in eq. (6), leads to

$$
E(r, \omega)=s_{0}\left(r, r_{0}, \omega\right) \cdot m_{0}(\omega)+S_{0}(r, \omega) \cdot \Lambda(\omega) \cdot A(\omega) \cdot S_{0}^{t}\left(r_{0}, \omega\right) \cdot m_{0}(\omega),
$$

where $\Lambda(\omega)$ is a block-diagonal matrix built from the dynamical dipolar polarizabilities associated with each microsystem

$$
\Lambda(\omega)=\left(\begin{array}{ccccc}
\alpha_{1}(\omega) & 0 & 0 & \cdots & 0 \\
0 & \alpha_{2}(\omega) & 0 & \cdots & 0 \\
0 & 0 & \alpha_{3}(\omega) & \cdots & 0 \\
0 & 0 & 0 & \cdots & \alpha_{n}(\omega)
\end{array}\right)
$$

and

$$
S_{0}\left(r_{0}, \omega\right)=\left\{s_{0}\left(r_{0}, r_{1}, \omega\right) ; s_{0}\left(r_{0}, r_{2}, \omega\right) ; \cdots ; s_{0}\left(r_{0}, r_{n}, \omega\right)\right\}
$$

In eq. (13), the response field $\boldsymbol{E}(\boldsymbol{r}, \omega)$ is linear with respect to the external dipole moment $\boldsymbol{m}_{0}(\omega)$. Consequently, the dyadic tensor connecting these two vectors is simply the field susceptibility of the composite system

$$
\mathbf{S}\left(r, r^{\prime}, \omega\right)=s_{0}\left(r, r^{\prime}, \omega\right)+S_{0}(r, \omega) \cdot \Lambda(\omega) \cdot A(\omega) \cdot S_{0}^{t}\left(r^{\prime}, \omega\right)
$$




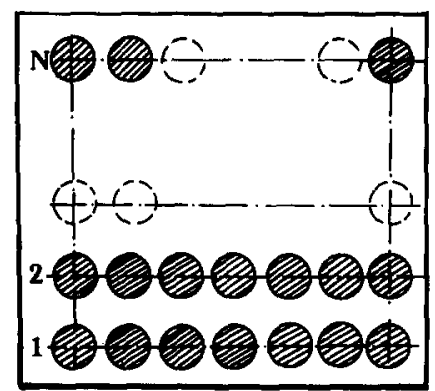

Fig. 2. Schematic illustration of a system composed of $(N)$ finite lines of physisorbed particles. As described in section 2.3 the field susceptibility associated with such a system can be derived by introducing a sequence of $(N)$ Dyson's equations.

Eq. (16) establishes a simple and compact relation between the field susceptibility of a reference system (for example, the free surface of a solid) and a more complex system composed of a finite number of particles interacting with the first one. Such a general equation is similar to Dyson's equation used in the electronic Green's function theory [16]. The many-body character of the interactions between each particle is included in the matrix product $\Lambda(\omega) \cdot A(\omega)$. Note that eq. (16) can be solved analytically for a single particle interacting with a perfectly plane substrate [17].

\subsection{A sequence of Dyson's equations for a large number of particles}

In the present description, working in the real space $\left\{\boldsymbol{r}_{i} ; \boldsymbol{r}_{j}\right\}$ with a spatially localized defect allows the self-consistent problem to be solved accurately. Nevertheless, for a large number of particles this opportunity is also associated with computational difficulties due to the large size of the self-consistent matrix $A(\omega)$. In such a situation, it seems more adequate to derive the field susceptibility $S$ of the whole system by introducing a sequence of Dyson's equations. The method consists of propagating the field susceptibility outside a growing ensemble of particles. In order to illustrate this procedure we consider a system composed of $(N)$ lines formed of $n$ atoms physisorbed on a surface (cf. fig. 2). According to eq. (16) the field susceptibility associated with the first line is given by

$$
S_{1}\left(r, r^{\prime}, \omega\right)=s_{0}\left(r, r^{\prime}, \omega\right)+S_{0}(r, \omega) \cdot \Lambda^{(1)}(\omega) \cdot A^{(1)}(\omega) \cdot S_{0}^{t}\left(r^{\prime}, \omega\right) .
$$

In this equation, the index (1) refers to the first line of the two-dimensional array described in fig. (2). In a similar way, the field susceptibility $S_{2}$ associated with the two first lines of particles can be deduced from the knowledge of $S_{1}$. This leads to

$$
S_{2}\left(r, r^{\prime}, \omega\right)=S_{1}\left(r, r^{\prime}, \omega\right)+S_{1}(r, \omega) \cdot A^{(2)}(\omega) \cdot A^{(2)}(\omega) \cdot S_{1}^{\mathrm{t}}\left(\boldsymbol{r}^{\prime}, \omega\right) .
$$

At this stage the matrix $\Lambda^{(2)}(\omega)$ is built from the polarizabilities of the particles belonging to the second line and $A^{(2)}(\omega)$ is obtained by replacing $s_{0}$ by $S_{1}$ into eq. (12). Thus, by repeating this procedure, it is possible to derive the field susceptibility $S_{N}$ of a discrete system formed of $(N)$ lines of particles from the field susceptibility $S_{N-1}$ of a system composed of $(N-1)$ ones. This leads to

$$
S_{N}\left(r, r^{\prime}, \omega\right)=S_{N-1}\left(r, r^{\prime}, \omega\right)+S_{N-1}(r, \omega) \cdot \Lambda^{(N)}(\omega) \cdot A^{(N)}(\omega) \cdot S_{N-1}^{\mathrm{t}}\left(\boldsymbol{r}^{\prime}, \omega\right) .
$$

Such an algorithm avoids the numerical inversion of a large matrix $(3 n N \times 3 n N)$. It reduces the self-consistent problem to a succession of small matrix $(3 n \times 3 n)$ inversions. This procedure is therefore relevant for all studies concerned with a great number of degrees of freedom because it is much less critical in terms of computer memory allocation. As a by-product it gives the field susceptibility of all single micro-systems added to the preceding configuration. This feature can be an asset when studying 
crystal growth processes as will be shown later. Consequently, it can provide an interesting basis for studying collective properties and the energy transfer occurring inside composite systems. Moreover, the use of this sequence of equations is not limited by numerical accuracy because the field susceptibility avoids the problem of self-energy.

\section{Application to many-body dispersive interactions in the vicinity of a surface}

One of the difficulties in formulating a theory for the dispersive van der Waals interactions between two systems lies in the fact that van der Waals effects have a many-body character [18-20]. For example, when a pair of atoms or molecules is placed near a surface, an additional contribution called substrate-mediated energy (SME) must be taken into account [21]. The influence of SME has been analyzed in the literature. It has been shown that this lateral effect plays a significant role in the structural properties of adsorbed overlayers. Moreover, in a slightly different context, many-body interactions give rise to a measurable increase of the long-range interaction between van der Waals clusters [22].

In this section, we discuss the efficiency of the present method for including such non-additive effects in the calculation of van der Waals dispersive energy inside a finite line of rare gas atoms physisorbed on surfaces of experimental interest. For a small number of physisorbed atoms the calculation can be performed by using the procedure described in section 2.2 in which the field susceptibility is derived from a single sequence of Dyson's equation.

\subsection{Dispersive energy inside a finite line of physisorbed atoms}

Let us consider a finite line composed of $(n)$ identical atoms located along the $O X$-direction at the positions $r_{i}=\left(X_{i}, 0, D\right)$. The field susceptibility $S$ of this system can be obtained from eq. (16) by set

$$
A(\omega)=\alpha(\omega) l
$$

where $\alpha(\omega)$ represents the dynamical polarizability of the physisorbed atoms and $l$ is the identity matrix.

Approaching a $(n+1)$ th atom labelled (a) at a distance $\boldsymbol{R}_{\mathrm{a}}$ from the system, gives rise to a dispersive energy $U\left(\boldsymbol{R}_{\mathrm{a}}\right)$. According to the van der Waals force theory [19], the knowledge of the tensor $S$ defined in eq. (16) is sufficient to detcrminc $U\left(\boldsymbol{R}_{\mathrm{a}}\right)$ (cf. eq. (1)). Note that the relation (1) is only restricted to the dipolar dispersive contribution. Nevertheless, higher order terms could easily be included in our formalism by introducing appropriate multipolar polarizabilities. After substituting eqs. (16) and (20) into eq. (1), this energy can be expressed as

$$
U\left(\boldsymbol{R}_{\mathrm{a}}\right)=U_{0}\left(\boldsymbol{R}_{\mathrm{a}}\right)+U_{1}\left(\boldsymbol{R}_{\mathrm{a}}\right),
$$

where the first term represents the van der Waals energy between the atom (a) and the isolated surface

$$
U_{0}\left(\boldsymbol{R}_{\mathrm{a}}\right)=-\frac{\hbar}{2 \pi} \int_{0}^{\infty} \operatorname{Tr}\left[\alpha_{\mathrm{a}}(\mathrm{i} u) \cdot \boldsymbol{s}_{0}\left(\boldsymbol{R}_{\mathrm{a}}, \boldsymbol{R}_{\mathrm{a}}, \mathrm{i} u\right)\right] \mathrm{d} u .
$$

The second term in eq. (21) accounts for different many-body effects with the substrate and the other physisorbed atoms. It is given by

$$
U_{1}\left(\boldsymbol{R}_{\mathrm{a}}\right)=-\frac{\hbar}{2 \pi} \int_{0}^{\infty} \alpha_{\mathrm{a}}(\mathrm{i} u) \alpha(\mathrm{i} u) \operatorname{Tr}\left\{S_{0}\left(\boldsymbol{R}_{\mathrm{a}}, \mathrm{i} u\right) \cdot A(\mathrm{i} u) \cdot S_{0}^{\mathrm{t}}\left(\boldsymbol{R}_{\mathrm{a}}, \mathrm{i} u\right)\right\} \mathrm{d} u .
$$


In this expression the matrix $A(\mathrm{i} u)$ accounts for multiple reflections between each atom inside the line and the substrate (cf. eqs. (10) and (11)). Since in this application the atoms lying on the surface are assumed to be identical, this matrix can be rewritten as

$$
A(\mathrm{i} u)=\{l-\alpha(\mathrm{i} u) C(\mathrm{i} u)\}^{-1}
$$

with

$$
C(\mathrm{i} u)=\left(\begin{array}{cccc}
s_{0}\left(r_{1}, r_{1}, \mathrm{i} u\right) & s_{0}\left(r_{1}, r_{2}, \mathrm{i} u\right) & \ldots & s_{0}\left(r_{1}, r_{n}, \mathrm{i} u\right) \\
s_{0}\left(r_{2}, r_{1}, \mathrm{i} u\right) & s_{0}\left(r_{2}, r_{2}, \mathrm{i} u\right) & \ldots & s_{0}\left(r_{2}, r_{n}, \mathrm{i} u\right) \\
\ldots & \ldots & \ldots & \ldots \\
\ldots & \ldots & \ldots & \ldots \\
s_{0}\left(r_{n}, r_{1}, \mathrm{i} u\right) & s_{0}\left(r_{n}, r_{2}, \mathrm{i} u\right) & \ldots & s_{0}\left(r_{n}, r_{n}, \mathrm{i} u\right)
\end{array}\right)
$$

For standard interatomic distances chosen beyond the repulsive regime, each matrix element of $\alpha(\mathrm{i} u) C(\mathrm{i} u)$ remains $\ll 1$. It is then possible to perform the following expansion

$$
A(\mathrm{i} u)=I+\sum_{s=1}^{\infty} \alpha^{(s)}(\mathrm{i} u) C^{(s)}(\mathrm{i} u),
$$

where $C^{(s)}(\mathrm{i} u)$ represents the matrix product of $s$ th order. Substituting this matrix expansion into the general expression (23) leads to

$$
U_{1}\left(\boldsymbol{R}_{\mathrm{a}}\right)=U_{1}^{0}\left(\boldsymbol{R}_{\mathrm{a}}\right)+\sum_{s=1}^{\infty} U_{1}^{(s)}\left(\boldsymbol{R}_{\mathrm{a}}\right),
$$

where the leading term of this expansion is just the well known additive van der Waals energy experienced by the probe atom in the presence of other atoms. The higher order contributions are given by

$$
U_{1}^{(s)}\left(\boldsymbol{R}_{\mathrm{a}}\right)=-\frac{\hbar}{2 \pi} \int_{0}^{\infty} \alpha_{\mathrm{a}}(\mathrm{i} u) \alpha^{(s+1)}(\mathrm{i} u) \operatorname{Tr}\left\{S_{0}\left(\boldsymbol{R}_{\mathrm{a}}, \mathrm{i} u\right) \cdot C^{(s)}(\mathrm{i} u) \cdot S_{0}^{\mathrm{t}}\left(\boldsymbol{R}_{\mathrm{a}}, \mathrm{i} u\right)\right\} \mathrm{d} u .
$$

This set of equations explicitly gives the contributions for three-body energy, four-body energy, etc., and converges very rapidly (cf. table 1). This treatment is general and can be applied to other problems than the one presented in this section (optical properties of molecular clusters, physical adsorption of fullerenes, etc.).

Table 1

Variation of the first many-body contributions (in meV) inside an ensemble of 15 layered Xe atoms deposited on an $\mathrm{Al}$ surface (cf. fig. 5)

\begin{tabular}{llll}
\hline$Z_{\mathrm{a}}(\AA)$ & Two-body energy & Three-body energy & Four-body energy \\
\hline 3.0 & -307.50 & 22.50 & -4.07 \\
3.4 & -263.91 & 13.88 & -3.34 \\
3.8 & -225.85 & 10.94 & -2.57 \\
4.2 & -189.94 & 8.22 & -2.03 \\
4.6 & -156.09 & 5.96 & -1.64 \\
5.0 & -125.75 & 4.14 & -1.31
\end{tabular}

We have considered the center site (c) of the pattern. The parameter $D$ of the lattice is equal to $4 \AA$, and $Z_{\mathrm{a}}$ represents the adsorption distance of the probe atom above its site. 


\subsection{Application to specific systems}

In order to illustrate the efficiency of the method, we present in this part a brief numerical application based on relations (22), (27) and (28) for a typical rare gas atom-substrate system. First, we define the different functions used to describe the atom and the surface.

\section{(i) The adsorbate}

In the context of the van der Waals interactions, the linear response properties of an isolated atom can be modeled with the following relation $[21,23]$

$$
\alpha_{a}(\mathrm{i} u)=\frac{\alpha_{\mathrm{a}}(0) \omega_{0}^{2}}{u^{2}+\omega_{0}^{2}},
$$

where the parameters $\alpha_{a}(0)$ and $\omega_{0}$ are defined in ref. [21]. For a xenon atom one has $\alpha_{a}(0)=27.11$ a.u. and $\omega_{0}=0.527$ a.u.

\section{(ii) The substrate}

Until now, the only approximation adopted here has been the dipolar approximation introduced to treat the dynamical response of the physisorbed particles. The different expressions of the dispersive energy given in previous sections have been obtained without restrictive assumptions about the description of the substrate. The specificity of the surface enters the eqs. (22), (23), (25) and (28) through the field susceptibility $s_{0}$. From results available in the literature $[9,10]$, the real profile of the surface and the non-local character of the response of the substrate could be included in our application without formal difficulties. However, for the sake of clarity, we will restrict our study to the case of a perfectly planar and local surface. In such a model the field susceptibility $s_{v}\left(r, r^{\prime}, \mathrm{i} u\right)$ can be expressed in terms of the local dielectric constant $\epsilon(\mathrm{i} u)$ of the material at imaginary frequency. In other words, the $k$-dependence of the dielectric constant of the solid has been neglected. In fact, as already discussed in the literature [24], this assumption leads to a slightly overestimated numerical value of the adsorption energy. Within this approximation one obtains

$$
s_{0}\left(r, r^{\prime}, \mathrm{i} u\right)=T\left(r, r^{\prime}\right)+s_{\mathrm{s}}\left(r, r^{\prime}, \mathrm{i} u\right), \text { if }\left(r \neq r^{\prime}\right)
$$

and

$$
s_{0}\left(r, r^{\prime}, \mathrm{i} u\right)=s_{\mathrm{s}}(r, r, \mathrm{i} u), \quad \text { if } \quad\left(r=r^{\prime}\right)
$$

where $T\left(r, r^{\prime}\right)$ labels the dipolar propagator in vacuum and $s_{\mathrm{s}}$ accounts for the presence of a surface. Note that these two different equalities express the fact that it is not possible to couple a given atom of the line with itself. Moreover, in absence of non-local effects in the substrate, the field susceptibility associated with the surface has a simple form because frequency and spatial dependence can be separated

$$
s_{\mathrm{s}}\left(r, r^{\prime}, \mathrm{i} u\right)=\Delta(\mathrm{i} u) F\left(r, r^{\prime}\right)
$$

where $\boldsymbol{F}$ is a dyadic tensor describing the spatial variation of $\boldsymbol{s}_{\mathrm{s}}$.

$$
\boldsymbol{F}\left(\boldsymbol{r}, \boldsymbol{r}^{\prime}\right)=\frac{1}{2 \pi} \int_{-\infty}^{\infty} \int_{-\infty}^{\infty} \frac{\mathrm{d} \boldsymbol{k}}{k} \boldsymbol{K} \boldsymbol{K}^{*} \exp \left[-\mathrm{i} \boldsymbol{k} \cdot\left(\boldsymbol{l}-\boldsymbol{l}^{\prime}\right)\right] \exp \left[-k\left(z+z^{\prime}\right)\right]
$$

with $K=(i k,-k), r=(l, z)$, and $r^{\prime}=\left(l^{\prime}, z^{\prime}\right)$. In eq. (32), $\Delta$ represents the non-retarded reflection coefficient of the surface at imaginary frequency

$$
\Delta(\mathrm{i} u)=\frac{[\epsilon(\mathrm{i} u)-1]}{[\epsilon(\mathrm{i} u)+1]} .
$$


Table 2

Numerical study of the influence of the substrate on the dispersion energy

\begin{tabular}{llc} 
& $U\left(\boldsymbol{R}_{\mathrm{a}}\right)$ & \\
\cline { 2 - 3 } & With substrate (meV) & Without substrate (meV) \\
\hline Site (a) & -179.56 & -60.63 \\
Site (b) & -209.17 & -92.52 \\
Site (c) & -239.29 & -124.94 \\
\hline
\end{tabular}

We have considered the three sites defined in fig. 5. The distance $D$ between two consecutive atoms is $4.25 \AA$.

Note that useful model formulae exist $[21,24]$ where one may assume a Lorentzian form to describe this reflection coefficient.

From these relations it is easy to obtain a very concise expression for the matrix $C(\mathrm{i} u)$ occurring in the expression (28) of the dispersion energy. This leads to

$$
C(\mathrm{i} u)=T+\Delta(\mathrm{i} u) F \text {, }
$$

with

$$
T(\omega)=\left(\begin{array}{cccc}
0 & \boldsymbol{T}\left(\boldsymbol{r}_{1}, \boldsymbol{r}_{2}\right) & \cdots & \boldsymbol{T}\left(\boldsymbol{r}_{1}, \boldsymbol{r}_{n}\right) \\
\boldsymbol{T}\left(\boldsymbol{r}_{2}, \boldsymbol{r}_{1}\right) & 0 & \cdots & \boldsymbol{T}\left(\boldsymbol{r}_{2}, \boldsymbol{r}_{n}\right) \\
\ldots & \cdots & \cdots & \cdots \\
\cdots & \cdots & \cdots & \cdots \\
T\left(\boldsymbol{r}_{n}, \boldsymbol{r}_{1}\right) & \boldsymbol{T}\left(\boldsymbol{r}_{n}, \boldsymbol{r}_{2}\right) & \cdots & 0
\end{array}\right)
$$

and

$$
F=\left(\begin{array}{cccc}
F\left(r_{1}, r_{1}\right) & F\left(r_{1}, r_{2}\right) & \ldots & F\left(r_{1}, r_{n}\right) \\
F\left(r_{2}, r_{1}\right) & F\left(r_{2}, r_{2}\right) & \ldots & F\left(r_{2}, r_{n}\right) \\
\ldots & \ldots & \ldots & \ldots \\
\ldots & \ldots & \ldots & \ldots \\
F\left(r_{n}, r_{1}\right) & F\left(r_{n}, r_{2}\right) & \ldots & F\left(r_{n}, r_{n}\right)
\end{array}\right)
$$

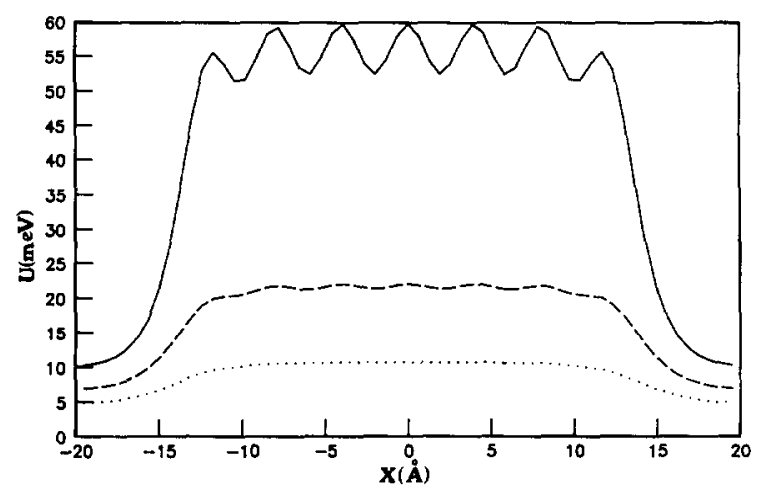

Fig. 3. Lateral variation of the attractive dispersion energy $U\left[\boldsymbol{R}_{\mathrm{a}}=\left(X_{\mathrm{a}}, 0, Z_{\mathrm{a}}\right)\right]$ between a xenon atom and a finite line of seven xenon atoms physisorbed on a perfectly plane $\mathrm{Al}$ surface. The interatomic distances between two consecutive atoms inside the line is chosen equal to $4 \AA$ and the atom-substrate separation is equal to $3 \AA$. The probe atom scans the system just above the line in the $X$ direction at a constant distance $Z_{\mathrm{a}}$ from the substrate: $\left(-\frac{-}{(} Z_{\mathrm{a}}=7 \AA ;(---): Z_{\mathrm{a}}=8 \AA ;(\cdots \cdots): Z_{\mathrm{a}}=9 \AA\right.$. The atomic corrugations recorded by the probe-atom progressively collapse when $Z_{\mathrm{a}}$ increases. 

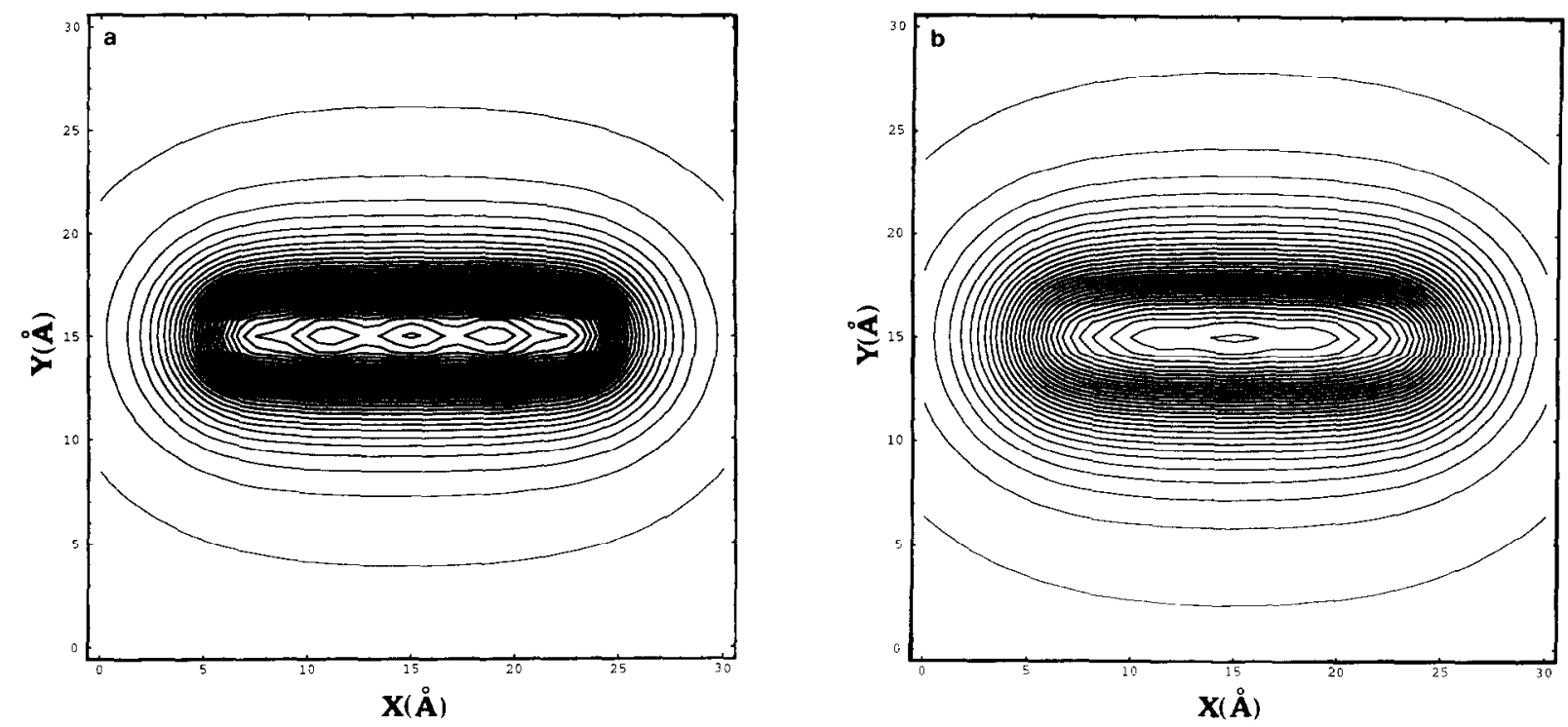

Fig. 4. Two sequences of iso-energy curves $U\left(\boldsymbol{R}_{\mathrm{a}}\right)=$ const. experienced by a xenon probe-atom above a line of five xenon atoms physisorbed on an Al surface. The geometrical parameters are the same as in fig. 3. The calculation is performed in a plane parallel to the surface $\left(Z_{\mathrm{a}}=\right.$ const.) and the scanned area is $30 \times 30 \AA^{2}$. (a) $Z_{\mathrm{a}}=8 \AA$; the energy $U\left(\boldsymbol{R}_{\mathrm{a}}\right)$ varies from 6.5 to 22.6 meV by steps of $0.53 \mathrm{meV}$, (b) $Z_{\mathrm{a}}=9 \AA$; $U\left(\boldsymbol{R}_{\mathrm{a}}\right)$ varies from 4.5 to $12 \mathrm{meV}$ by steps $0.25 \mathrm{meV}$.

Relations (35) to (37) can then be used to study numerically the variation of the potential $U\left(\boldsymbol{R}_{\mathrm{a}}\right)$ defined in eqs. (21), (22) and (23). As an example, we have considered the Xe-Al system. The parameters $\alpha_{\mathrm{a}}$ and $\omega_{0}$ and the function $\Delta(\mathrm{i} u)$ used to describe the atoms and the substrate are found in ref. [21]. For aluminium, the two parameters used to build a Lorentzian representation of $\Delta(\mathrm{i} u)$ are given in table 2 of ref. [21].

In fig. 3 , the variation of the attractive dispersion energy between a Xe probe-atom and a line of seven physisorbed $\mathrm{Xe}$ atoms is plotted as a function of the lateral position $X_{\mathrm{a}}$ of the probe-atom. The interatomic distance between two consecutive atoms inside the line is chosen equal to $4 \AA$ and the scanned distance is $40 \AA$. As expected, one notices that the atomic corrugations experienced by the probe-atom depend dramatically on the approach distance $Z_{\mathrm{a}}$. For $Z_{\mathrm{a}}=7 \AA$ the van der Waals energy modulation detected above the line reaches about $8 \mathrm{meV}$, while it vanishes beyond $9 \AA$. This behavior is consistent with the localized character of the interatomic van der Waals forces and explains the origin of the difficulty to image individual atoms in atomic-force microscopy working in the attractive mode. Finally, let us recall that the three curves of fig. 3 have been obtaincd through application of the whole many-body approach described in section 3.1. If only the two-body interaction energy term is retained in the calculation, the amplitude of the modulation of the upper curve decreases by about $7.5 \%$.

Fig. 4 presents two series of equipotential curves $U\left(\boldsymbol{R}_{\mathrm{a}}\right)=$ const. These curves have been obtained by calculating the variation of the dispersion energy between a Xe probe-atom and a line composed of five $\mathrm{Xe}$ atoms physisorbed on an $\mathrm{Al}$ surface. The calculation is performed in a plane parallel to the surface at the distance $Z_{\mathrm{a}}=$ const. and the scanned area is $30 \times 30 \AA^{2}$. As illustrated in fig. $4 \mathrm{a}$, for a distance of $8 \AA$ from the surface, the three atoms located at the center of the line can easily be recognized in the van der Waals energy pattern. But around $9 \AA$ (fig. $4 \mathrm{~b}$ ), this information is lost and in this case only the central atom can be distinguished. Moreover, in all cases the energy distribution appears to be strongly localized around the atomic structures. 


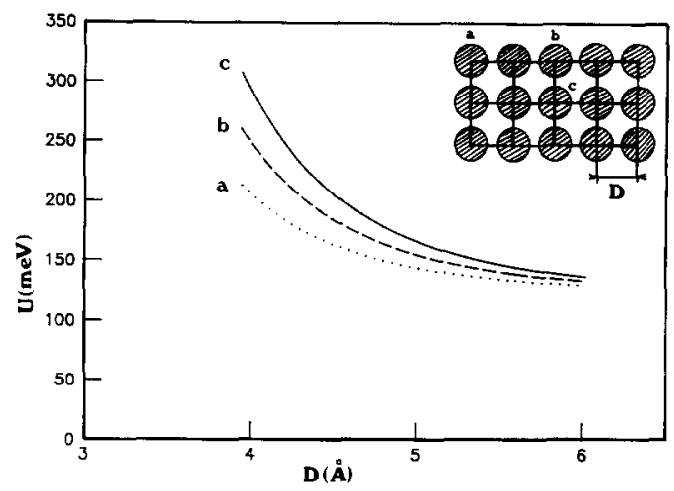

Fig. 5. Variation of the van der Waals binding energy inside an ensemble of 15 layered Xe atoms deposited on an Al surface. We have considered three different sites. (a) Corner site (the atom has two nearest neighbours), (b) site along an edge line (the atom has three nearest neighbours), (c) center site (four nearest neighbours).

We now address the question of the van der Waals binding energy inside an atomic array of physisorbed atoms. This quantity can easily be derived from the present formalism. In fact it suffices to calculate the energy $U\left(\boldsymbol{R}_{\mathrm{a}}\right)$ between a Xe atom and the $(n-1)$ other atoms of the array. In this case, the vector $\boldsymbol{R}_{\mathrm{a}}$ represents the location of a selected atom inside the aggregate. Let us notice that the present formalism systematically eliminates the cohesion energy due to the interactions between the $(n-1)$ remaining atoms. In this last application (cf. fig. 5), we consider a rectangular aggregate composed of 15 atoms organized on the surface at the nodes of a square lattice of period $D$. We then calculate the binding energy $U\left(\boldsymbol{R}_{\mathrm{a}}\right)$ as a function of $D$ for different non-equivalent sites - labelled (a), (b) and (c) - of the probe-atom. We can see on fig. 5 that the three curves associated with the different sites asymptoticaly tend to the same value $(\simeq 130 \mathrm{meV})$ which corresponds to the physisorption energy of an isolated atom. As expected, the van der Waals energy is maximum on the central site and minimum near the corner site. Thus, for an equilibrium interatomic distance equal to $4.25 \AA$ between two consecutive $\mathrm{Xe}$ atoms, the van der Waals binding energy varies between 230 and $175 \mathrm{meV}$ when the number of nearest neighbours varies between 2 and 4. As described in section 3.1, as well as in ref. [20], the total van der Waals energy between two systems can be decomposed into many-body interaction terms. In table 1, we have evaluated each of the first leading terms of the series for the system described in fig. 5. We have considered the variation of the three first terms when the probe atom is located above the site (c). It may be seen that the series converges very rapidly. This behavior is consistent with the relative weak value of the rare gas atom polarizability. The two-body term represents about $92 \%$ of the total energy. Another interesting point has to be addressed. It concerns the influence of the substrate on the magnitude order of the dispersion energy. In table 2 we show how the dispersion energy experienced by the atoms located at the sites (a), (b) and (c), varies when the calculation is done with or without any substrate.

\section{Conclusion and discussion}

In this paper we have described a unified framework for studying the dynamical response of a composite system in the vicinity of solid surfaces. Assuming the response of the naked substrate is known via a certain field susceptibility, we have proposed a new algorithm leading to a general description of the response of the substrate when it is covered by a great number of particles. The method consists of 
propagating the field susceptibility outside a growing ensemblc of particles deposited on a surface. Such a procedure avoids the usual computer memory allocation difficulties arising with matrices of large size. As illustrated with the applications given in section 3, the present approach seems to be an efficient tool for modeling the response properties of nanometer scale structures lying on a surface and could also be a promising technique for describing the many-body effects and light-matter coupling that occur in scanning probe microscopy. Indeed, the same formalism as developed in this paper may be applied to the electromagnatic field: we simply need to substitute an incident plane wave to the dipolar fluctuation $\boldsymbol{m}_{0}(\omega)$ to be able to enter the domain of near field optics. Particularly, it will be useful to describe the non-radiative optical energy transfer in systems of complex geometry like those used in scanning near-field optical microscopy. In systems where a theoretical description of the electromagnetic field needs to couple a great number of elementary cells, the sequence of Dyson's equations described in section 2 offers an interesting opportunity to derive the optical field distribution over a large scale. Such a study is in progress [25].

\section{Acknowledgement}

The authors express their sincere thanks to Dr. J.P. Vigneron for a number of stimulating and interesting discussions.

\section{References}

[1] A.D. McLachlan, Proc. R. Soc. London A 274 (1963) 80.

[2] A.D. McLachlan, Mol. Phys. 7 (1964) 381.

[3] B. Linder, Adv. Chem. Phys. 12 (1967) 225.

[4] B. Linder and D.A. Rabenold, Adv. Quantum Chem. 6 (1972) 203.

[5] G.S. Agarwal, Phys. Rev. A 11 (1975) 230.

[6] H. Metiu, J. Chem. Phys. 76 (1982) 1765.

[7] H. Metiu, Prog. Surf. Sci. 17 (1984) 153.

[8] H. Metiu and P. Das, Annu. Rev. Phys. Chem. 35 (1984) 507.

[9] C. Girard, Phys. Rev. B 45 (1992) 1800, and references therein.

[10] C. Girard and C. Girardet, J. Chem. Phys. 86 (1987) 6581.

[11] C. Girard and D. Courjon, Phys. Rev. B 42 (1990) 9340.

[12] A. Dereux, J.P. Vigneron, Ph. Lambin and A.A. Lucas, Physica B 175 (1991) 65; C. Girard, X. Bouju and A. Dereux, in: Near Field Optics, NATO series E, Ed. D.W. Pohl, in press.

[13] D.M. Eigler and E.K. Schweizer, Nature 344 (1990) 524.

[14] J.A. Stroscio and D.M. Eigler, Science 254 (1991) 1319.

[15] C. Girard and X. Bouju, J. Chem. Phys. 95 (1991) 2056.

[16] E.N. Economou, Green's Functions in Quantum Physics, 2nd. ed. (Springer, Berlin, 1983).

[17] C. Girard, Pure Appl. Opt. A 1 (1992) 157.

[18] D. Langbein, Theory of van der Waals Attraction, Springer Tracts in Modern Physics (Springer, Berlin, 1974).

[19] J. Mahanty and B.W. Ninham, Dispersion Forces (Academic Press, London, 1976).

[20] A.A. Lucas, Physica 35 (1967) 353; M. Schmeits and A.A. Lucas, Prog. Surf. Sci. 14 (1983) 1.

[21] S. Rauber, J.R. Klein, M.W. Cole and L.W. Bruch, Surf. Sci. 123 (1982) 173.

[22] J. Cao and B.J. Berne, J. Chem. Phys. 97 (1992) 8628.

[23] G. Vidali and M.W. Cole, Surf. Sci. 110 (1981) 10.

[24] X.P. Jiang, F. Togio and M.W. Cole, Surf. Sci. 145 (1984) 281; 148 (1984) 21.

[25] O.J.F. Martin, A. Dereux and C. Girard, to be published. 